\title{
Confirmatory Factor Analysis of the Portuguese Version of the Arousal Predisposition Scale
}

\author{
Daniel Ruivo Marques $^{1,2}$ • Ana Allen Gomes ${ }^{3,4}$ - Maria Helena Pinto de Azevedo ${ }^{5}$
}

(C) Springer Science+Business Media New York 2017

\begin{abstract}
The Arousal Predisposition Scale (APS) is an instrument aimed to assess individual differences in selfreported arousability, specifically physiologically arousal. Besides, it is a scale frequently used in stress and sleeprelated researches. The principal purpose of this study is to determine the factor structure of the APS through confirmatory factor analysis (CFA) and to test the goodness-of-fit of oneand two-factor models. A sample of 341 undergraduate medical students of both genders aged 17 to 25 years was enrolled. Beyond the APS, the participants also filled out the Portuguese versions of the Pre-Sleep Arousal Scale (PSAS), Ford Insomnia Response to Stress Test (FIRST) and the Neuroticism scale of Eysenck Personality Inventory (EPI) for concurrent validity purposes. From the studied solutions, a modified two-factor model $(\mathrm{F} 1=$ emotional reactivity and F2 = trait anxiety) achieved the better fit to the data. The Portuguese version of the APS was also significantly correlated with measures of neuroticism, insomnia, sleep reactivity to
\end{abstract}

Daniel Ruivo Marques

drmarques@ua.pt

1 Department of Education and Psychology, University of Aveiro, Campus Universitário de Santiago, 3810-193 Aveiro, Portugal

2 Institute for Biomedical Imaging and Life Sciences, IBILI, Azinhaga de Santa Comba, 3000-548 Coimbra, Portugal

3 Faculty of Psychology and Educational Sciences, University of Coimbra, Rua do Colégio Novo, 3000-115 Coimbra, Portugal

4 CINEICC - Centro de Investigação do Núcleo de Estudos e Intervenção Cognitivo-Comportamental / Research \& Development Unit (FCT): Cognitive and Behavioural Center for Research and Intervention, Coimbra, Portugal

5 Faculty of Medicine, University of Coimbra, Rua Larga, 3004-504 Coimbra, Portugal stress, and cognitive and somatic arousal. The two-factor modified model appears to display a superior fit to the data, supporting a scoring model of the APS according to two different factors as suggested by a previous research on the Portuguese version. This study supports reliability and validity indicators of the APS and its use in research and applied settings.

Keywords Arousal predisposition scale $\cdot$ Confirmatory factor analysis · Validation · Psychometrics · Undergraduates

\section{Introduction}

Arousability is a construct intimately related to both psychological disorders and sleep disturbances/insomnia (Bonnet and Arand 2010; Dinzeo et al. 2004; Fernández-Mendoza et al. 2010; Miller et al. 2015). In insomnia, for example, it is consensual the idea of a generalized hyperarousal encompassing cognitive, affective, behavioral, physiological, and neurobiological systems (Riemann et al. 2010). Additionally, this increased level of activation seems to constitute a trait variable which is considered as one of the key predisposition factors to insomnia (Perlis et al. 2011; Harvey et al. 2014). Even so, it should be noted that there are instruments intended to evaluate arousal within a sleep / insomnia context and other measures aimed to assess general arousal.

The Arousal Predisposition Scale (APS) is a short and easy-to-administer scale composed by 12 items and specifically developed to evaluate physiological arousal. The original studies on APS demonstrated adequate psychometric properties (Coren and Mah 1993; Coren 1988, 1990). Moreover, the APS has been widely used in insomnia research (Fernández-Mendoza et al. 2010; LeBlanc et al. 2009). The scale enables responses in a Likert-type format with 5 options. 
However, in the European Portuguese version of the scale, the response options were slightly modified encompassing only 4 options (i.e., "never / almost never" and "seldom" were compiled into a single option labelled "almost never" since the existence of both original options were not quite discriminative in the Portuguese sample) (Marques et al. 2016a, b).

Despite the substantial using of the APS in research, there are few studies on its psychometric properties. Indeed, only the original works on the APS present some kind of psychometrics. In addition, data on structure composition of the APS is also lacking. To the best of our knowledge, there are no studies using confirmatory factor analysis (CFA) focusing on this scale.

The aforementioned study carried out recurring to a European Portuguese sample of undergraduate medical students found adequate reliability as assessed by internal consistency $(\alpha=.85)$ and good convergent and criterion validity indicators (Marques et al. 2016a, b). Moreover, the authors performed an exploratory factor analysis (EFA) and found a two-factor structure. One factor was named "emotional reactivity" (comprising items 2, 3, 4, 6, 8, 9, 10, 11, 12) and the other was labeled "trait anxiety" (comprising items 1, 5, and 7). To our knowledge, this was the first study with known data positing a different and alternative factorial composition for the APS. Usually, the scoring of the APS is done summing all the 12 items' scores producing a total result. This new proposed two-factor structure may prove to be very useful in research and subsequently in clinical settings as arousability is a relevant personality trait present in most psychological disorders (Dinzeo et al. 2004). Moreover, new conceptualizations about the APS (i.e., two-factors) may contribute to parsimony of measures in future studies. That is, when it is essential to evaluate trait anxiety, researchers may use one of the APS subscales rather than longer trait anxiety questionnaires such as State-Trait Anxiety Inventory (STAI) (Marques et al. 2016a, b). Finally, as the APS is so much used worldwide, studies on its properties that justifies or not its inclusion on specific research protocols are needed.

Specifically, regarding insomnia, one should note that arousability is a relevant construct in comprehensive models of insomnia such as the sleep-interfering and sleepinterpreting processes model (Lundh and Broman 2000). Several studies have consistently shown that arousability is a predictor of sleep disturbance/insomnia (Marques et al. 2016c; Marques et al. 2016a, b). In addition, previous research showed that arousability is intimately associated with sleep reactivity which in recent years demonstrated to be a relevant factor to insomnia's development and maintenance (Marques et al. 2016d). In this line, it is surprising that there are no psychometric studies on the APS. Hence, the aim of the current study is to further contribute to validation of the European Portuguese version of the APS recurring to structural equation modeling-SEM (specifically CFA) in order to test the adjustment of the exploratory factorial structure proposed by Marques et al. (2016a, b) in an independent sample of Portuguese undergraduate students.

\section{Methods}

\section{Participants}

In this study, a total of 341 medical students from a Portuguese public university were recruited (219 women; mean age $=19.31 ; S D=1.21 ;$ range $17-23$ years). One of the participants did not indicate the gender. Comparing mean ages by gender, no statistical difference was found $\left[t_{(338)}=-1.565\right.$; $p=.119$ ). Only students from the first three years of medicine course were enrolled (1st year $=31.4 \%$, 2nd year $=31.1 \%$ and 3rd year $=37.5 \%$ ). Pertaining to marital status all individuals were single. One should note that the undergraduate sample of medical students used in the present study is independent from the one used by Marques et al. (2016a, b).

\section{Measures}

\section{Arousal Predisposition Scale (APS)}

The Arousal Predisposition Scale (APS) comprises 12 items intended to assess vulnerability to arousal, specifically physiological arousal. Each item is scored on a 4-point scale. Higher score denotes higher arousability predisposition (Coren and Mah 1993). In the current study we used the European Portuguese version by Marques et al. (2016a, b) which have shown adequate reliability and validity indicators.

\section{Eysenck Personality Inventory (EPI)}

The short version of the EPI (EPI-12, Eysenck and Eysenck 1964) is a 12-item tool to assess Neuroticism and Extraversion dimensions of personality. In the current work, only Neuroticism (NE) - 6 items - was used. The item 12 "I suffer from sleeplessness" was removed from the computation of this dimension, as it might constitute a confounding variable. The Cronbach's alpha was .61. In the current study we used the European Portuguese version by Silva et al. $(1994,1995)$ which was developed within a larger research project on the adaptation into European Portuguese of Standard Shiftwork Index (SSI).

\section{Self-Reported Insomnia}

Self-reported insomnia was assessed with item 12 (i.e., "I suffer from sleeplessness/insomnia") from the EPI-12 which is scored from $1=$ almost never to $4=$ almost always. 


\section{Pre-Sleep Arousal Scale (PSAS)}

The PSAS contains 16 items, each rated on a 5-point scale that describes symptoms of arousal at bedtime (Nicassio et al. 1985). Eight items evaluates cognitive arousal and eight evaluates somatic arousal. Higher scores suggest higher pre-sleep arousal. In the current study, the Cronbachs' alphas for somatic and cognitive arousal were .81 and .77 , respectively. In the current study we used the European Portuguese version by Azevedo et al. (2010) which showed adequate properties.

\section{Ford Insomnia Response to Stress Test (FIRST)}

The FIRST is a self-report scale designed to assess sleeprelated 'reactivity' i.e., the tendency to exhibit pronounced sleep disturbance in response to a stress challenge. Greater scores denote higher vulnerability to stress-related sleep disturbance (Drake et al. 2004). In the current study, the Cronbach's alpha was .80 . We used the European Portuguese version developed by Marques et al. (2016c) which showed good psychometric indicators for a youngadult sample.

\section{Procedure}

This study was approved by the Ethics Committee and the Scientific Council of the Faculty of Medicine of the University of Coimbra. The professors were initially contacted in order to obtain authorization to administer the questionnaires to the students at the beginning/ending of a class session (out of the evaluation period). The aims of the study were explained to the students, it was emphasized that their cooperation was voluntary, and confidentiality was ensured. All participants accepted to collaborate in the study and all ethics requirement were met.

\section{Statistical Analyses}

The IBM SPSS Statistics ${ }^{\mathrm{TM}}$ v.20 software was used to calculate descriptive statistics such as means, standard deviations, and skewness and kurtosis coefficients. Besides, Correlations matrices, corrected item-total corrected correlations, Cronbach's alphas, and Cronbach's alpha when item is deleted were also computed (Field, 2013). Independent samples $t$-tests were used to study differences between men and women. To examine the goodness-of-fit of different factor models, the AMOS Graphics ${ }^{\mathrm{TM}}$ software v.20 was used. All the analyses were performed according to Maximum Likelihood Estimator Type. Beforehand, important assumptions on data were analyzed: minimum sample size, absence of multicollinearity and singularity (examined through R-matrices), normality of data (examined through univariate kurtosis and skewness $[s k$ $<|3|$ and $k u<|7|]$ and standard Mardia's coefficients to check multivariate normality) and outliers (Mahalanobis $D^{2}$ distances) (Kline, 2005).

To compare the adjustment of the different models, the most cited goodness-of-fit indicators were used, namely the Chi-Square test $\left(\chi^{2}\right)$, the Chi-Square Critical Ratio $\left(\chi^{2} / \mathrm{df}\right)$, the Goodness-of-Fit Index (GFI), the Comparative Fit Index (CFI), and the Root Mean Square Error of Approximation (RMSEA). Moreover, to compare nested models, the Expected Cross Validation Index (ECVI), and Akaike's Information Criterion (AIC) were also computed (Kline, 2005). Modification Indices (MI > 11) were taken into consideration to perform post-hoc modifications. Besides, only associations among errors were considered. To be considered adequate, the models have to fulfil the following criteria: $p$ value of $\chi^{2}>.05 ; \chi^{2} / \mathrm{df} \leq 5 ; \mathrm{GFI} \geq .90 ; \mathrm{CFI} \geq .90$ and RMSEA $\leq .08$. Both ECVI and AIC should be as small as possible (Byrne 2010; Gores and Weston 2006). For evaluation of local adjustment standardized coefficients paths $(\lambda)>.40$ were considered adequate, as recommended by Tabachnick and Fidell (2012). Additionally, composite reliability (CR) - which is a new alternative to Cronbach's alpha taking into account measurement errors - and the average variance extracted (AVE) - a indicator of convergent validity - were computed (Byrne 2010; Hair et al. 2009). Values of $\mathrm{CR} \geq .7$ and $\mathrm{AVE} \geq .5$ are usually considered adequate values. For all analyses, $p$ values $<.05$ were considered statistically significant.

\section{Results}

\section{Descriptive Statistics}

Table 1 presents the main descriptive statistics pertaining to the APS. The mean ranged from 1.91 (item 12) to 2.42 (item 4). The skewness values ranged from .14 (item 8 ) to .59 (item 12). The kurtosis values ranged from - .13 (item 3) to - .64 (item 8 ). Both skewness and kurtosis values were within the recommended values for assuming univariate normality (i.e., $s k<|3|$ and $k u<|7|)$.

\section{Testing for One-Factor Model}

When the one-factor model was tested, that is, the one comprising a single latent variable regressed onto all the twelve items, the global adjustment was considered poor (cf. Table 2). Pertaining to the local adjustment the standardized coefficients paths ranged between .37 (item 12) and .75 (item 5). In terms of composite reliability (CR) the value was .85. In SEM, when one perform CFA, it is recommended to report AVE values as a convergent validity indicator. Convergent validity in SEM refers to the degree that a latent variable is explained by its observed variables. In our study, the AVE was .33 which is well below the recommended value of .50 . 
Table 1 Descriptive statistics concerning APS-PT items

\begin{tabular}{|c|c|c|c|c|}
\hline Items & M & SD & Skewness & Kurtosis \\
\hline 1. I am a calm person & 2.05 & .76 & .30 & -.36 \\
\hline 2. I get flustered if I have several things to do at once & 2.32 & .81 & .27 & -.36 \\
\hline $\begin{array}{l}\text { 3. Sudden changes of any kind produce an immediate emotional effect } \\
\text { on me }\end{array}$ & 2.21 & .82 & .36 & -.13 \\
\hline $\begin{array}{l}\text { 4. Strong emotions carry over for one or two hours after I leave the } \\
\text { situation which caused them }\end{array}$ & 2.42 & .86 & .19 & -.60 \\
\hline 5. I am restless and fidgety & 1.99 & .84 & .44 & -.55 \\
\hline 6. My mood is quickly influenced by entering new places & 2.18 & .82 & .27 & -.47 \\
\hline 7. I get excited easily & 2.08 & .88 & .47 & -.51 \\
\hline $\begin{array}{l}\text { 8. I find that my heart keeps beating fast for a while after I have been } \\
\text { "stirred up" }\end{array}$ & 2.24 & .84 & .14 & -.64 \\
\hline $\begin{array}{l}\text { 9. I can be emotionally moved by what other people consider to be } \\
\text { simple things }\end{array}$ & 2.02 & .88 & .51 & -.52 \\
\hline 10. I startle easily & 1.92 & .93 & .74 & -.33 \\
\hline 11. I am easily frustrated & 2.13 & .89 & .42 & -.56 \\
\hline $\begin{array}{l}\text { 12. I tend to remain excited or moved for a long period of time after } \\
\text { seeing a good movie }\end{array}$ & 1.91 & .85 & .59 & -.44 \\
\hline
\end{tabular}

M, Mean; SD, standard deviation

\section{Testing for Two-Factor Model}

When the two-factor model was tested, that is, the one comprising a two correlated latent variables regressed onto nine and three items, respectively, the global adjustment was considered fair (cf. Table 2). Pertaining to the local adjustment the standardized coefficients paths ranged between .37 (item 12) and .75 (item 5). In terms of CR, for factor 1 (emotional reactivity) the value was .93. Concerning AVE, the value was .33. For factor 2 (trait anxiety) the values were $\mathrm{CR}=.81$ and $\mathrm{AVE}=.60$. The correlation between both factors was $r=.70$.

\section{Testing for a Modified Two-Factor Model}

When the modified two-factor model was tested, that is, the one comprising three estimated additional residual covariances (between items 2 and 3, items 4 and 8, and items 9 and 12) suggested both by the modification indices values and theory, the global adjustment was considered very good. In terms of CR, for factor 1 (emotional reactivity) the value was .80. Concerning AVE, the value was .31. For factor 2 (trait anxiety) the values were $\mathrm{CR}=.81$ and $\mathrm{AVE}=.60$. The correlation between factors was $r=.71$. In overall, according to the recommendations presented by Byrne (2010), it may be concluded that our model achieved a good fit to the data (Fig. 1).

\section{Reliability Analysis}

Cronbach's alpha concerning Portuguese APS version total score was .85. The corrected item-total corrected correlations ranged from .37 (item 12) to .66 (item 5) ensuring homogeneity of the items. With respect to Cronbach's alpha when item is deleted we observed that the exclusion of any item does not improve the overall internal consistency of the scale. Regarding the two factors, the Cronbach's alphas were .81 for "emotional reactivity" factor and .80 for "trait anxiety" factor.

\section{Association of the APS with Other Measures}

In Table 3 are displayed the Pearson's correlation coefficients for the associations among the APS total score and its factors with other relevant variables. Overall, it was found moderate correlations among the Portuguese APS version total score and its factors (i.e., emotional reactivity and trait anxiety) and insomnia, neuroticism, sleep reactivity to stress, and cognitive and somatic arousal $(p<.001)$.
Table 2 Goodness-of-fit indices of the various tested models

\begin{tabular}{lcrlllll}
\hline Models & \multicolumn{1}{l}{ Х2 $(\mathrm{df})$} & \multicolumn{1}{c}{ 2 $/ \mathrm{df}$} & RMSEA [CI 90\%] & CFI & GFI & ECVI & AIC \\
\hline Model 1 & $549.15(54)^{*}$ & 10.170 & $.11[.62-.84]$ & .80 & .86 & .87 & 597.15 \\
Model 2 & $278.30(53)^{*}$ & 5.521 & $.07[.07-.08]$ & .91 & .93 & .48 & 328.30 \\
Model 2a & $175.212(50)^{*}$ & 3.504 & $.06[.05-.07]$ & .95 & .96 & .33 & 231.21 \\
\hline
\end{tabular}

$* p<.001 ; \mathrm{df}$, degrees of freedom; RMSEA, Root Mean square error of approximation; CFI, Comparative Fit index; GFI, Goodness-of-fit index; ECVI, Expected Cross validation index; AIC, Akaike's Information criterion 
Fig. 1 Factor loadings in the two-factor modified model (Final Model). The displayed loadings represent the standardized coefficient patterns

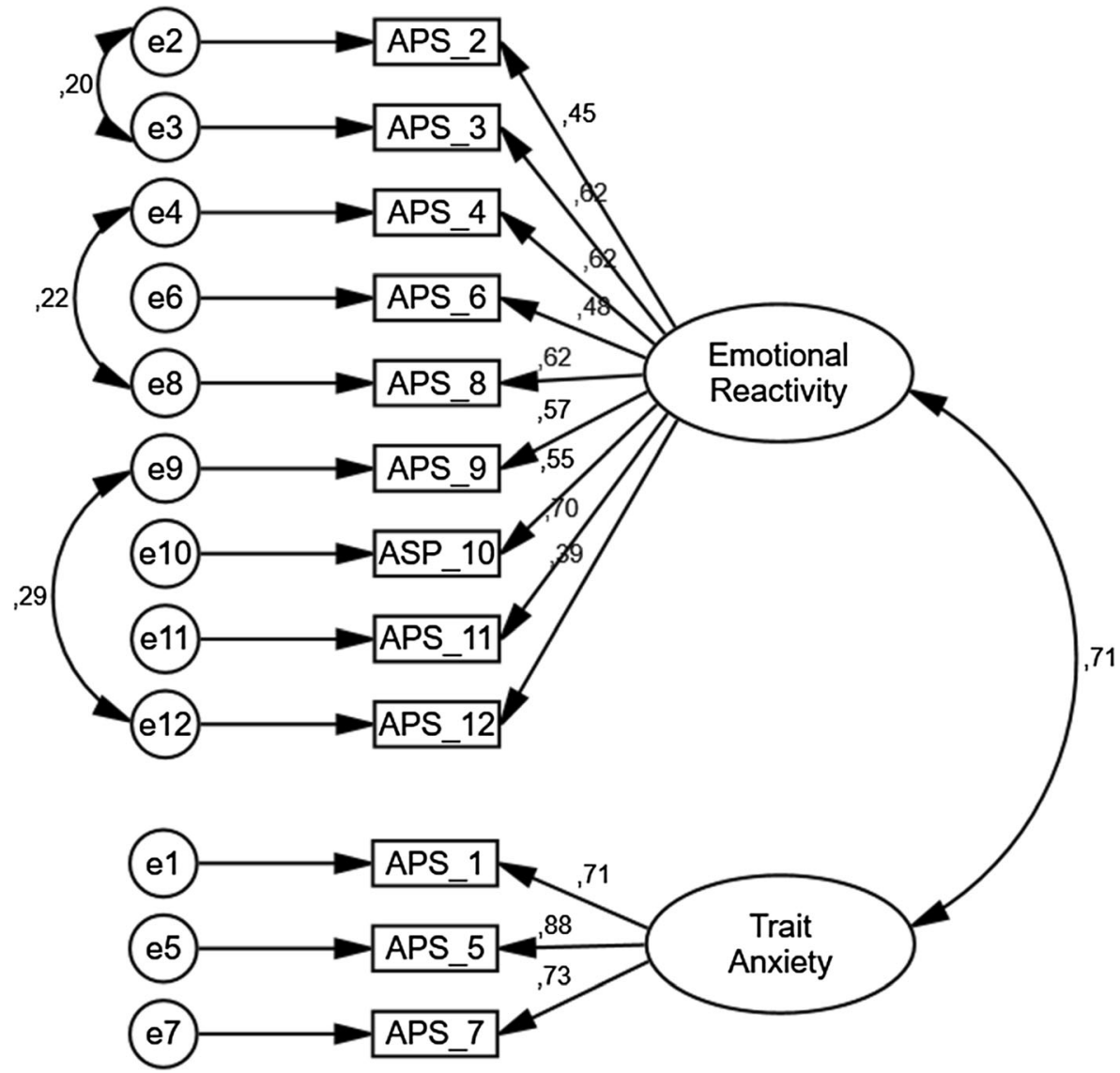

\section{Discussion}

In the current study, our major aim was to test through a CFA the best factorial solution for the APS-PT. Overall, our results enhance the good psychometric properties of a European Portuguese version of the APS to assess arousability. Additionally, the two-factor structure of the APS previously presented in a work by Marques et al. (2016a, b) is also confirmed here through confirmatory factor analysis. These findings adds evidence to the idea that the European Portuguese version of the APS seems to measure two constructs

Table 3 Correlation matrix among the APS, EPI, FIRST and PSAS scores

\begin{tabular}{llll}
\hline & $\begin{array}{l}\text { APS } \\
r\end{array}$ & $\begin{array}{l}\text { APS (F1) } \\
r\end{array}$ & $\begin{array}{l}\text { APS (F2) } \\
r\end{array}$ \\
\hline Neuroticism (EPI-12) & $.58^{* *}$ & $.55^{* *}$ & $.50^{* *}$ \\
Insomnia_item 12 (EPI) & $.28^{* *}$ & $.26^{* *}$ & $.27^{* *}$ \\
Sleep reactivity to stress (FIRST) & $.51^{* *}$ & $.50^{* *}$ & $.42^{* *}$ \\
Pre-sleep cognitive arousal (PSAS) & $.40^{* *}$ & $.39^{* *}$ & $.34^{* *}$ \\
Pre-sleep somatic arousal (PSAS) & $.38^{* *}$ & $.35^{* *}$ & $.35^{* *}$ \\
\hline
\end{tabular}

$* * p<.001$

APS (F1), Emotional reactivity; APS (F2), Trait anxiety associated with general arousability: emotional reactivity and trait anxiety. Despite the satisfactory fit achieved with the twofactor model, we tested if the model could be improved with some post-hoc modifications. This revised model was based upon the modification indices suggested by the software. Only error correlations among items belonging to the same factor were enabled (Byrne 2010). When the errors between items 2 and 3,4 and 8, and 9 and 12 were correlated, the model achieved very good goodness-of-fit indices (both CFI and GFI > .95) according to Byrne (2010). This association among errors is a common practice in CFA and may be due to a similar phrasing of the items. For instance, both item 4 "Strong emotions carry over for one or two hours after I leave the situation which caused them" and item 8 "I find that my heart keeps beating fast for a while after I have been "stirred up" "concern to the persistence of emotional activity. Even so, one should be aware that there is always the possibility that these post-hoc modifications result from idiosyncratic features of the studied sample. However, even considering this hypothesis, the superiority and suitability of the two-factor structure seems convincing. Unfortunately, there are no systematic studies on the psychometrics of the APS which enable us to compare our results. Notwithstanding, the aforementioned study by Marques et al. (2016a, b) suggests that perhaps the current using of the APS might be underestimated since this 
scale seems to evaluate two different (albeit related) dimensions of arousability.

Despite these encouraging findings some limitations should be outlined. First, the sample's nature, that is, the convenience sample constituted by undergraduate students which may compromise the generalization of the results. Second, the absence of a physiological arousal measure to correlate with our self-report measure of arousability. Finally, it was not possible to perform a test-retest study, thus, we cannot assure the temporal stability of the APS.

For future research it is essential to study the psychometric properties of the APS in other countries/cultures in order to understand the invariance of the factorial composition we found. Besides, it would be important to further examine the contribution of arousability components to insomnia's predisposition. Also, it would be very useful to enroll clinical samples with diverse diagnoses and explore the APS psychometrics. Finally, as the APS is a common measure in psychophysiological studies, researchers should attempt to correlate physiological indicators such as the electroencephalography or evoked potentials with the self-reported arousability - either with the APS general arousability measure or its dimensions.

In conclusion, it was presented evidence showing that the Portuguese version of the APS comprises two related factors. It is our aim to continue exploring the psychometric characteristics of the APS and we incite researchers worldwide to disseminate their findings on APS properties.

Acknowledgements The co-operation of the professors and students is gratefully acknowledged.

\section{Compliance with Ethical Standards}

Conflict of Interest The authors declare that they have no conflict of interest.

Human and Animal Rights This article does not contain any studies with human participants or animals performed by any of the authors.

Informed Consent Informed consent was obtained from all individual participants included in the study.

\section{References}

Azevedo, M. H., Maia, B., Marques, M., Bos, S., Nogueira, V., Soares, M. J., et al. (2010). Psychometric properties of the Portuguese version of the pre-sleep arousal scale. Journal of Sleep Research, 19(Suppl. 2), e157.

Bonnet, M., \& Arand, D. (2010). Hyperarousal and insomnia: State of the science. Sleep Medicine Reviews, 14(1), 9-15. doi:10.1016/j. smrv.2009.05.002.
Byrne, B. (2010). Structural equation modeling with AMOS: Basic concepts, applications, and programming (2nd ed.). New York: Routledge.

Coren, S. (1988). Prediction of insomnia from arousability predisposition scores: Scale development and cross-validation. Behaviour Research and Therapy, 26(5), 415-420. doi:10.1016/0005-7967(88)90076-9.

Coren, S. (1990). The arousal predisposition scale: Normative data. Bulletin of the Psychonomic Society, 28(6), 551-552. doi:10.3758 /BF03334078.

Coren, S., \& Mah, K. (1993). Prediction of physiological arousability: A validation of the arousal predisposition scale. Behaviour Research and Therapy, 31(2), 215-219. doi:10.1016/0005-7967(93)90076-7.

Dinzeo, T., Cohen, A., Nienow, T., \& Docherty, N. (2004). Stress and arousability in schizophrenia. Schizophrenia Research, 71(1), 127135. doi:10.1016/j.schres.2004.01.008.

Drake, C., Richardson, G., Roehrs, T., Scofield, H., \& Roth, T. (2004). Vulnerability to stress-related sleep disturbance and hyperarousal. Sleep, 27(2), 285-291.

Eysenck, H. J., \& Eysenck, S. G. (1964). Manual of the Eysenck personality Inventory. London: University of London Press.

Fernández-Mendoza, J., Vela-Bueno, A., Vgontzas, A., Ramos-Pláton, M., Olavarrieta-Bernardino, S., Bixler, E., et al. (2010). Cognitiveemotional hyperarousal as a premorbid characteristic of individuals vulnerable to insomnia. Psychosomatic Medicine, 72(4), 397-403. doi:10.1097/PSY.0b013e3181d75319.

Field, A. (2013). Discovering statistics using IBM SPSS statistics (4th ed.). London: Sage Publications.

Gores, P., \& Weston, R. (2006). A brief guide to structural equation modeling. The Counseling Psychology, 34(5), 719-751. doi:10.1177/0011000006286345.

Hair, J., Black, W., Babin, B., \& Anderson, R. (2009). Multivariate data analysis (7th ed.). New Jersey: Prentice Hall.

Harvey, C., Gehrman, P., \& Espie, C. (2014). Who is predisposed to insomnia: A review of familial aggregation, stress-reactivity, personality and coping style. Sleep Medicine Reviews, 18(3), 237-247. doi:10.1016/j.smrv.2013.11.004.

Kline, R. (2005). Principles and practice of structural equation modeling (2nd ed.). New York: Guilford.

LeBlanc, M., Mérette, C., Savard, J., Ivers, H., Baillargeon, L., \& Morin, C. (2009). Incidence and risk factors of insomnia in a populationbased sample. Sleep, 32(8), 1027-1037.

Lundh, L., \& Broman, J. (2000). Insomnia as an interaction between sleepinterfering and sleep-interpreting processes. Journal of Psychosomatic Research, 49(5), 299-310. doi:10.1016/S0022-3999(00)00150-1.

Marques, D., Gomes, A. A., \& Azevedo, M. H. (2016a). Portuguese version of the arousal predisposition scale (APS). Journal of Sleep Research, 25(Suppl. 1), e159. doi:10.1111/jsr.12446.

Marques, D., Gomes, A. A., \& Azevedo, M. H. (2016b). Portuguese version of the arousal predisposition scale: Preliminary evidence for a two-factor structure in a nonclinical sample. Manuscript submitted for publication

Marques, D., Gomes, A., Ferreira, M., \& Azevedo, M. H. (2016c). Don't worry, sleep well: Predictors of sleep loss over worry. Sleep and Biological Rhythms, 14(3), 309-318. doi:10.1007/s41105-0160060-Z.

Marques, D., Gomes, A., Drake, C., Roth, T., \& Azevedo, M. H. (2016d). Assessing stress-induced sleep reactivity in college students: The European Portuguese version of the Ford insomnia response to stress test (FIRST). Behavioral Sleep Medicine. doi:10.1080 /15402002.2016.1210151.

Miller, C., Kyle, S., Gordon, C., Espie, C., Grunstein, R., Mullins, A., et al. (2015). Physiological markers of arousal change with psychological treatment for insomnia: A preliminary investigation. PloS One, 10(12), e0145317. doi:10.1371/journal.pone.0145317.

Nicassio, P. M., Mendlowitz, D. R., Fussell, J. J., \& Petras, L. (1985). The phenomenology of the pre-sleep state: The development of the pre- 
sleep arousal scale. Behaviour Research Therapy, 23(3), 263-271. doi:10.1016/0005-7967(85)90004-X.

Perlis, M., Shaw, P., Cano, G., \& Espie, C. (2011). Models of insomnia. In M. Kryger, T. Roth, \& W. Dement (Eds.), Principles and Practice of Sleep Medicine (5ed.) (pp. 850-865). Elsevier Saunders: Missouri.

Riemann, D., Spiegelhalder, K., Feige, B., Voderholzer, U., Berger, M., Perlis, M., \& Nissen, C. (2010). The hyperarousal model of insomnia: A review of the concept and its evidence. Sleep Medicine Reviews, 14(1), 19-31. doi:10.1016/j.smrv.2009.04.002.
Silva, C. F., Azevedo, M. H., \& Dias, M. R. (1994). Estudo Padronizado do Trabalho por Turnos. Versao Experimental. Instituto de Psicologia Médica da Faculdade de Medicina: Universidade de Coimbra.

Silva, C. F., Azevedo, M. H. P., \& Dias, M. R. (1995). Estudo padronizado do trabalho por turnos - versão portuguesa do SSI. Psychologica, 13, 27-36.

Tabachnick, B., \& Fidell, L. (2012). Using multivariate statistics (6th ed.). Boston: Pearson Education. 